\title{
Olanzapine versus fosaprepitant for the prevention of concurrent chemotherapy radiotherapy-induced nausea and vomiting
}

\author{
Rudolph M Navari, MD, PhD, ${ }^{\text {ab }}$ Cindy K Nagy, RN, ${ }^{\text {a }}$ Jennifer Le-Rademacher, $\mathrm{PhD},{ }^{\mathrm{c}}$ and \\ Charles L Loprinzi, MDc
}

${ }^{a}$ Department of Medicine Indiana University School of Medicine South Bend, Indiana; ${ }^{b}$ University of Notre Dame, Notre Dame, Indiana; and 'Department of Medical Oncology, Mayo Clinic, Rochester, Minnesota

\begin{abstract}
Background Concurrent chemotherapy radiation therapy may result in significant nausea and vomiting. There have been few studies reporting effective interventions for preventing treatment-related nausea and vomiting.

Objective To compare olanzapine with fosaprepitant for the prevention of nausea and vomiting in patients receiving concurrent highly emetogenic chemotherapy (HEC) and radiotherapy for locally advanced head and neck or esophageal cancer.

Methods 120 chemotherapy and radiotherapy naïve patients with head and neck cancer who were receiving concurrent local radiation and cisplatin were randomized to receive either olanzapine or fosaprepitant in combination with palonosetron and dexamethasone for the prevention of chemotherapy- and radiation-induced nausea and vomiting. The olanzapine, palonosetron, dexamethasone (OPD) regimen was $10 \mathrm{mg}$ oral olanzapine, $0.25 \mathrm{mg}$ IV palonosetron, and $20 \mathrm{mg}$ IV dexamethasone before chemotherapy on day 1 , and $10 \mathrm{mg} /$ day of oral olanzapine before chemotherapy on days $2-4$. The fosaprepitant, palonosetron, dexamethasone (FPD) regimen was $150 \mathrm{mg}$ IV fosaprepitant, $0.25 \mathrm{mg}$ IV palonosetron, and $12 \mathrm{mg}$ IV dexamethasone before chemotherapy on day 1 , and $4 \mathrm{mg}$ dexamethasone PO BID, before chemotherapy days 2 and 3 .

Results 101 of the 120 patients were evaluable. In 51 patients who received the OPD regimen, the complete response (CR; no emesis, no rescue medication) rate was $88 \%$ for the acute period ( $24 \mathrm{~h}$ after chemotherapy), $76 \%$ for the delayed period (days $2-5)$, and $76 \%$ for the overall period $(0-120 \mathrm{~h})$. In 50 patients who received the FPD regimen, the CR was $84 \%$ acute, $74 \%$ delayed, and $74 \%$ overall $(P>.01$ for all periods). Patients with no nausea $(0$, on a scale $0-10$, visual analogue scale) were: OPD: $86 \%$ acute, $71 \%$ delayed, $71 \%$ overall; FPD: $78 \%$ acute, $40 \%$ delayed, $40 \%$ overall $P>.01$ for acute; $P<.01$ for delayed and overall) There were no grade 3 or 4 toxicities.
\end{abstract}

Conclusions CR was similar for OPD and FPD; nausea in the delayed and overall periods was significantly improved with OPD compared with FPD $(P<.01)$.

Funding Reich Endowment for the Care of the Whole Patient.

$\mathrm{C}$ hemotherapy-induced nausea and vomiting $(\mathrm{CINV})$ is associated with a significant deterioration in patient quality of life and is perceived by patients as a major adverse effect of the treatment. ${ }^{1}$ There have been significant improvements in recent years in the control of chemotherapy-induced emesis with the use of the 5-hydroxytryptamine-3 (5-HT3) receptor antagonists, ${ }^{2,3}$ dexamethasone, ${ }^{2,3}$ the neurokinin-1(NK-1) receptor antagonists, ${ }^{4-6}$ and olanzapine, an antipsychotic that blocks multiple neurotransmitters in the central nervous system..$^{7-9}$ Chemotherapy-induced nausea, however, has not been as well controlled and remains a significant clinical problem. ${ }^{8-10}$

Radiation-induced nausea and vomiting (RINV) also has a significant adverse effect on life qual- ity. ${ }^{11-13}$ Few reported clinical trials have provided effective prevention or treatment recommendations for RINV. ${ }^{14-16}$ Concurrent chemotherapy radiotherapy is commonly used for locally advanced head and neck cancers ${ }^{17}$ and locally advanced esophageal cancers. ${ }^{18}$ There are no known prospective phase 3 clinical trials for the prevention of chemotherapy radiotherapy-induced nausea and vomiting for these malignancies. Published guidelines ${ }^{15}$ suggest that antiemetic prophylaxis for chemotherapy radiotherapy treatment should be based on the emetogenicity of the chemotherapy.

The purpose of this randomized, double-blind phase 3 study was to compare the efficacies of olanzapine and fosaprepitant, each combined with palonosetron and dexamethasone for the prevention of

Accepted for publication February 8, 2016. Correspondence: Rudolph M Navari; rmnavari@gmail.com.Disclosures: The authors have no conflicts of interest. JCSO 2016;14:141-147. (02016 Frontline Medical Communications. doi: 10.12788/ jeso.0245. 
nausea and vomiting in patients with locally advanced head and neck cancers and locally advanced esophageal cancers who were receiving highly emetogenic chemotherapy (HEC) concurrently with radiotherapy.

\section{Methods}

\section{Patient selection}

Eligible patients were aged 18 years or older, had histologically or cytologically confirmed locally advanced head and neck cancer or locally advanced esophageal cancer, and were chemotherapy and radiotherapy naïve. They were scheduled to receive HEC (cisplatin $>70 \mathrm{mg} / \mathrm{m}^{2}$ and 5-fluorouracil, $750 \mathrm{mg} / \mathrm{m}^{2}$ a day for 4 days) and concurrent radiotherapy (60-70 Gy, daily equal fractions, 5 days a week, for 6-7 weeks). The patients were treated at 3 outpatient oncology treatment centers with medical oncologists and radiation oncologists at each site. They were studied only during their first course of chemotherapy.

\section{Inclusion and exclusion criteria}

The inclusion criteria were: no nausea or vomiting in the 24 hours before beginning chemotherapy; serum creatinine, $<2.0 \mathrm{mg} / \mathrm{dL}$ (normal, 1-2 mg/dL); serum bilirubin, $<2.0 \mathrm{mg} / \mathrm{dL}$ (normal 1-2 mg/dL); AST or ALT, <3 times upper limits of normal; absolute neutrophil count, $>1500 \mathrm{~mm}^{3}$ (normal, 1500-5000 mm $\mathrm{mm}^{3}$ ); no severe cognitive compromise; no known history of CNS disease (eg, brain metastases, seizure disorder); no treatment with another antipsychotic agent for 30 days before or during protocol therapy; no chronic phenothiazine administration as an antipsychotic agent, no concurrent use of amifostine; no concurrent abdominal radiotherapy; no concurrent use of quinolone antibiotic therapy; no chronic alcoholism; no known hypersensitivity to olanzapine; no known uncontrolled cardiac arrhythmia, uncontrolled congestive heart failure, or acute myocardial infarction within the previous 6 months; and no history of diabetic ketoacidosis or uncontrolled diabetes mellitus. Patients of childbearing potential had to consent to use adequate contraception throughout protocol therapy and have had a negative urine pregnancy test. Patients were excluded if they had lost $>10 \%$ of normal body weight over 3 months, were not able to take oral medications, or had a feeding tube.

\section{Informed consent}

All of the patients gave written informed consent, and the study was approved by the institutional review committees of each participating site.

\section{Study design and treatment regimen}

Eligible patients were randomized to either olanzapine, palonosetron, and dexamethasone (OPD) or fosaprepitant, palonosetron, dexamethasone (FPD) before their first course of chemotherapy. The randomization was performed according to a computer-generated random assignment schedule. All of the patients were receiving daily radiotherapy and had completed 2 weeks of the scheduled 6-7 weeks of local radiotherapy. Patients were further stratified according to sex and to disease (head and neck cancer or esophageal cancer).

All patients who received the OPD regimen received, on the day of chemotherapy, day 1, dexamethasone $20 \mathrm{mg}$ IV and palonosetron, $0.25 \mathrm{mg}$ IV, 30-60 minutes before chemotherapy administration. Patients also began olanzapine $10 \mathrm{mg}$ PO on the day of chemotherapy (day 1 ) and continued $10 \mathrm{mg}$ PO daily for days 2-4 after chemotherapy.

Patients who received the FPD regimen received on day 1, dexamethasone $12 \mathrm{mg} I V$, palonosetron $0.25 \mathrm{mg} \mathrm{IV}$, and fosaprepitant $150 \mathrm{mg} \mathrm{IV,} \mathrm{30-60} \mathrm{minutes} \mathrm{before} \mathrm{chemo-}$ therapy. After chemotherapy, patients received oral dexamethasone $4 \mathrm{mg}$ BID on days 2-3. Patients were permitted to take rescue therapy of the treating investigator's choice for nausea and/or emesis/retching based on clinical circumstances. Patients who required rescue therapy were permitted to continue on the study at the discretion of the treating investigator in consultation with the patient.

The trial arms in the study were blinded. Patients in the OPD arm received intravenous placebo before chemotherapy (in lieu of fosaprepitant) and oral placebo BID for days 2-3 (in lieu of dexamethasone). Patients in the FPD arm received oral placebo (in lieu of olanzapine) on days 1 to 4 .

\section{Study visits and assessment procedures}

In the prestudy period, all pertinent demographics (age, sex, height, weight) and medical data (site and stage of disease, Eastern Cooperative Oncology Group [ECOG] performance status rating, laboratory values, and medications) were recorded. The MD Anderson Symptom Inventory (MDASI $)^{19}$ was used to measure key symptoms daily for the entire study period. The use of the MDASI in this study was to determine if there were any toxicities related to the antiemetic regimens.

Beginning with the first day of chemotherapy (day 1) and daily through day 5, patients were asked to record daily episodes of vomiting/retching (number and time), the daily intensity of symptoms using the MDASI, and the use of rescue therapy. Patients were also asked to record daily degrees of nausea using a visual analogue scale from $0-10$, with 0 indicating no nausea and 10 indicating a maximal level of nausea. A nurse/research coordinator contacted each patient daily (days 2-5) to remind the patient to complete forms and to query toxicities.

\section{Statistical methods}

The primary endpoint in the study was complete response (CR; no emetic episodes and no use of rescue medication) for the overall period (0-120 hours after chemotherapy). Secondary endpoints were CR in the acute phase (0-24 
hours after chemotherapy) and delayed phase (days 2-5 after chemotherapy), and no nausea (0 on a scale of $0-10$, visual analogue scale) in the acute, delayed, and overall periods. The study was powered to detect a $25 \%$ increase in CR rate in the OPD arm compared with the FPD arm. Assuming 15\% CR in the FPD arm, 50 evaluable patients per arm would provide $80 \%$ power to detect a change of this magnitude at a 2 -sided type 1 error of 0.05 . The total number of patients enrolled was elevated to account for a $10 \%$ dropout.

Demographic data and patient characteristics were summarized using median (range) for continuous variables and frequency (percentage) for categorical variables. The frequencies of severe toxicities and adverse events were calculated. The percentages of patients with $\mathrm{CR}$ and the percentage of patients with no nausea (Visual Analogue Scale score 0 ) for the acute period, the delayed period, and the overall periods were estimated along with $95 \%$ confidence intervals (CIs). The proportions of patients with CR and the proportions of patients without nausea were compared between the treatment arms.

The mean MDASI symptom scores over days 1-5 were calculated. A repeated measures analysis of variance was performed to compare symptom scores during the 5 days after chemotherapy. Seventeen analyses of variance were performed, so the level of significance was lowered to 0.01 as an adjustment for multiple comparisons.

\section{Results}

The distribution and randomization of the study patients is shown in Figure 1. In all, 120 patients were assessed for eligibility; 2 were excluded because they had nausea in the previous 24 hours, leaving 118 patients to be randomized. One patient in each group did not receive the allocated intervention after randomization. Seven patients in each group did not complete the allocated intervention. For the OPD group, 3 patients declined to complete the planned course of chemotherapy, 2 patients did not complete the 4 days of olanzapine because of missed doses, and 2 did not receive the planned daily radiotherapy during the study period. For the FPD group, 3 patients declined to complete the planned course of chemotherapy, 3 patients did not complete the 2 days of dexamethasone because of missed doses, and 1 did not receive the planned radiotherapy during the study period. One patient in the FPD arm was excluded from analysis because of incomplete data. Patient characteristics are presented in Table 1 for the 101 patients who completed the assigned antiemetic regimen and provided complete follow-up data.

\section{Primary efficacy parameters}

The CR rates for the acute period, the delayed period, and the overall period in 51 patients receiving the OPD regimen and for 50 patients receiving the FPD regimen are shown in Figure 2. There were no significant differences $(P$ $>.05)$ in the complete responses between the OPD regimen and the FPD regimen, for the acute, delayed or overall periods.

The 6 patients in the OPD group who did not have a $\mathrm{CR}$ in the acute period required rescue because of emesis. Twelve patients in the OPD group did not have a CR in

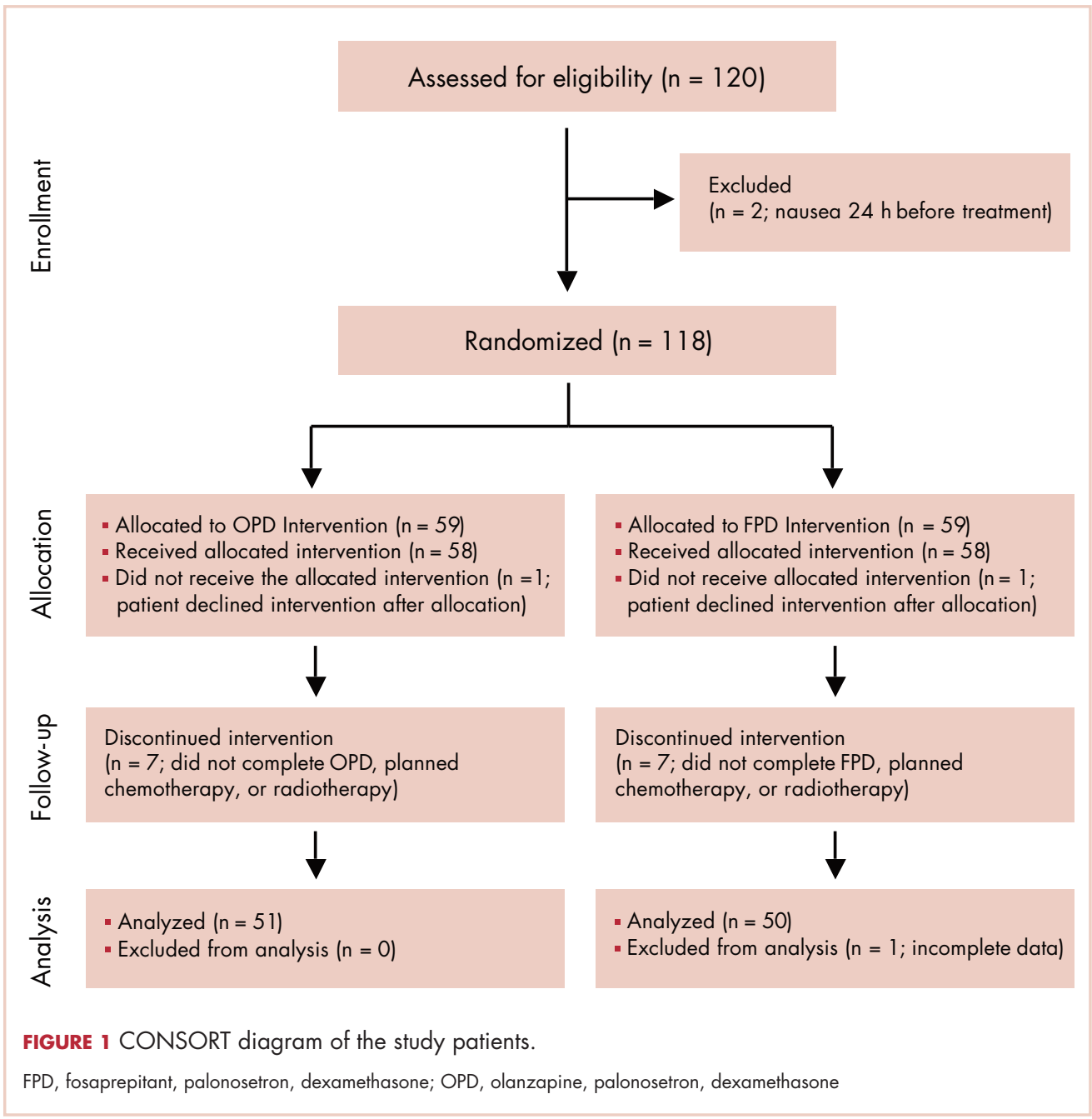




\begin{tabular}{|c|c|c|}
\hline Characteristic & $\begin{array}{l}\text { OPD } \\
\text { regimen }\end{array}$ & $\begin{array}{l}\text { FPD } \\
\text { regimen }\end{array}$ \\
\hline No. of patients & 51 & 50 \\
\hline Median age, y (range) & $63(59-76)$ & $61(52-71)$ \\
\hline Sex - women, $n(\%)$ & $11(22)$ & $12(24)$ \\
\hline \multicolumn{3}{|l|}{ ECOG, n (\%) } \\
\hline 0 & $31(61)$ & $33(66)$ \\
\hline 1 & $10(20)$ & $9(18)$ \\
\hline 2 & $10(20)$ & $8(18)$ \\
\hline \multicolumn{3}{|l|}{ Diagnosis, $\mathrm{n}(\%)$} \\
\hline Head and neck & $36(71)$ & $34(68)$ \\
\hline Esophageal & $15(29)$ & $16(32)$ \\
\hline \multicolumn{3}{|l|}{ Initial measurements (mean) } \\
\hline Height, in. (range) & $68(60-75)$ & $69(61-76)$ \\
\hline Weight, lb (range) & $\begin{array}{c}145 \\
(110-220)\end{array}$ & $\begin{array}{c}149 \\
(105-229)\end{array}$ \\
\hline $\begin{array}{l}\text { Body mass index, } \mathrm{kg} / \mathrm{m} 2 \\
\text { (range) }\end{array}$ & $23(21-26)$ & $23(20-27)$ \\
\hline \multicolumn{3}{|c|}{$\begin{array}{l}\text { ECOG, Eastern Cooperative Oncology Group; FPD, fosaprepitant, } \\
\text { palonosetron, dexamethasone; OPD, olanzapine, palonosetron, } \\
\text { dexamethasone }\end{array}$} \\
\hline
\end{tabular}

the delayed period. Nine had emesis on days 2 and 3 and all required rescue. Two had emesis on day 4 and one received rescue. One patient had emesis without rescue on day 5 .

The eight patients in the FPD group who did not have a $\mathrm{CR}$ in the acute period all had emesis and six required rescue in the first 24 hours after chemotherapy. There were 13 patients in the FPD group who did not have a CR in the delayed period. On day 2, 9 patients had emesis and 5 received rescue, and 4 patients had emesis without rescue. On day 3, 2 patients had emesis with rescue, and 1 patient had rescue without emesis. On day 4, 1 patient had rescue without emesis.

The control of nausea for the acute period, the delayed period, and the overall period in 51 patients receiving the OPD regimen and in 50 patients receiving the FPD regimen is shown in Figure 3. There were 7 patients in the OPD group who experienced nausea $(>0$, scale $0-10$, visual analogue scale) in the acute period. The 15 patients in the OPD group who experienced nausea in the delayed period consisted of 9 on day 2, 4 on day 3, and 2 on day 4. Eleven patients in the FPD group had nausea in the acute period. The occurrence of nausea in the delayed period for the FPD group was 15 patients on day 2, 8 patients on day 3, 3 on day 4 , and 4 on day 5 .

There was no significant difference $(P=.28)$ for the control of nausea between the OPD regimen and the FPD regimen for the acute period but there were significant

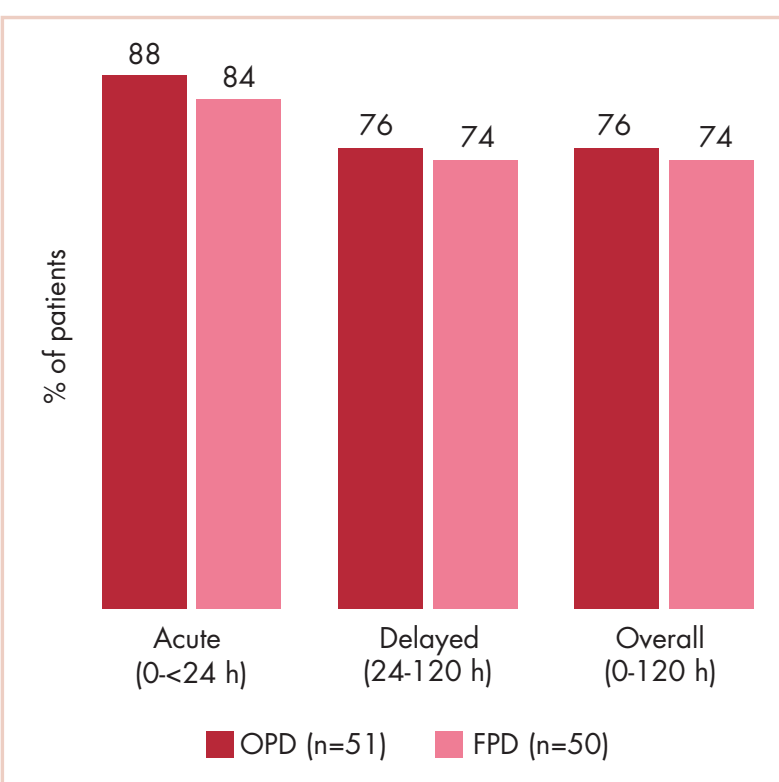

FIGURE 2 Percentage of patients with a complete response (no emetic episodes and no use of rescue medication) for those receiving concurrent highly emetogenic chemotherapy and radiotherapy. P not significant for acute, delayed, and overall.

FPD, fosaprepitant, palonosetron, dexamethasone; OPD, olanzapine, palonosetron, dexamethasone

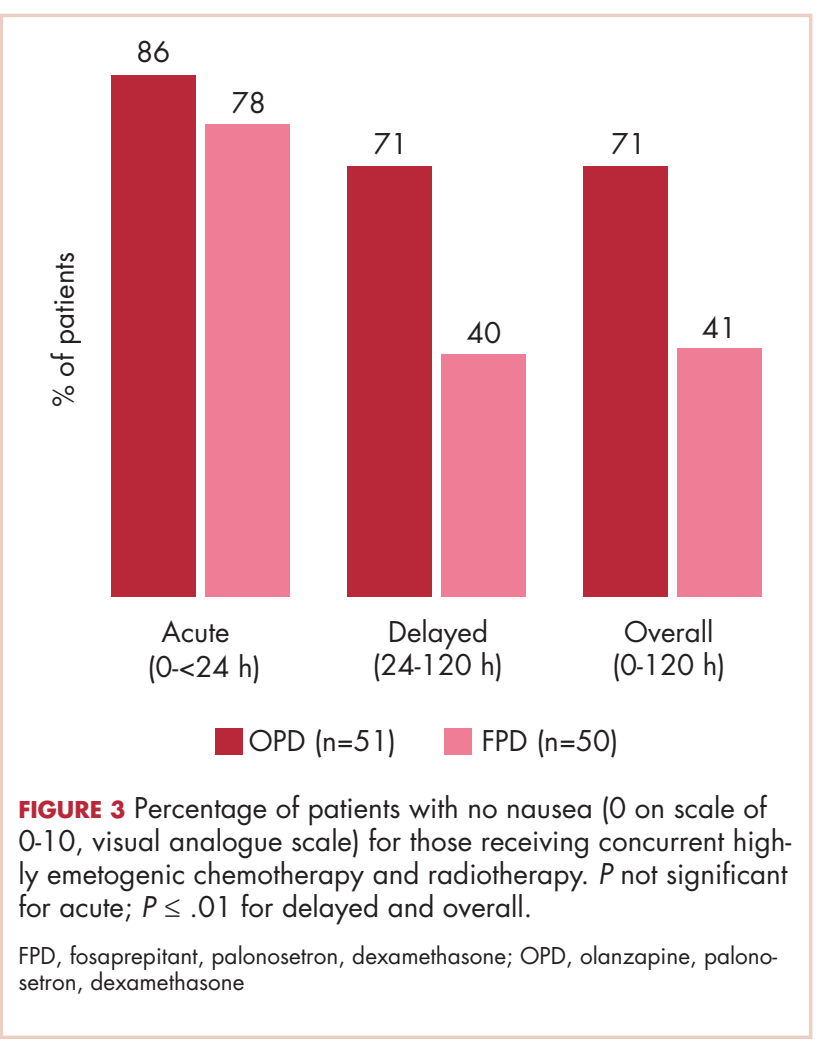

differences $(P=.001)$ between the OPD regimen and the FPD regimen for the delayed and overall periods.

An additional analysis including all 118 patients ran- 


\begin{tabular}{|c|c|c|c|c|c|c|c|c|c|c|}
\hline Symptom & Day $1^{a}$ & Day 2 & Day 3 & Day 4 & Day 5 & Day $1^{a}$ & Day 2 & Day 3 & Day 4 & Day 5 \\
\hline Pain & 1.3 & 1.6 & 1.3 & 1.5 & 1.5 & 1.2 & 1.3 & 1.5 & 1.2 & 1.5 \\
\hline Fatigue & 3.9 & 4.0 & 4.3 & 4.2 & 4.1 & 3.1 & 3.1 & 3.3 & 3.2 & 3.3 \\
\hline Problems remembering & 1.3 & 1.1 & 1.4 & 1.5 & 1.5 & 2.0 & 2.0 & 2.3 & 1.9 & 2.1 \\
\hline Shortness of breath & 1.7 & 1.8 & 2.0 & 1.9 & 2.1 & 2.6 & 2.1 & 2.6 & 2.4 & 2.4 \\
\hline Lack of appetite & 1.7 & 2.0 & 2.2 & 1.8 & 1.7 & 2.1 & 2.5 & 2.0 & 2.3 & 1.8 \\
\hline Physical numbness & 0.9 & 1.3 & 1.1 & 1.4 & 1.1 & 2.0 & 2.3 & 1.9 & 1.8 & 1.9 \\
\hline General activityd $^{d}$ & 2.3 & 2.4 & 2.3 & 2.1 & 2.0 & 2.0 & 2.4 & 2.2 & 2.5 & 2.2 \\
\hline Mood & 0.8 & 1.1 & 0.9 & 1.1 & 1.1 & 1.9 & 1.9 & 1.9 & 1.8 & 1.8 \\
\hline Worke & 2.5 & 2.5 & 2.7 & 2.3 & 2.9 & 2.9 & 3.4 & 2.9 & 3.1 & 2.8 \\
\hline Relationships & 1.9 & 1.6 & 2.1 & 2.2 & 1.8 & 1.8 & 1.5 & 1.3 & 1.8 & 1.7 \\
\hline Walking & 1.7 & 1.7 & 2.3 & 2.8 & 2.0 & 2.2 & 1.9 & 2.4 & 2.1 & 2.0 \\
\hline Enjoyment & 1.7 & 2.3 & 2.2 & 1.9 & 2.2 & 3.0 & 2.9 & 2.5 & 2.8 & 2.6 \\
\hline
\end{tabular}

FPD, fosaprepitant, palonosetron, dexamethasone; MDASI, MD Anderson Symptom Inventory; OPD, olanzapine, palonosetron, dexamethasone

${ }^{a}$ Day 1 data represents a baseline, obtained before chemotherapy and antiemetic therapy. ${ }^{b} P<.01$ (comparison of day 2 score vs day 1 score in OPD arm). ${ }^{c} P<.01$ (comparison of day 2 scores between arms). ${ }^{d}$ Normal activity for the individual. e Deskwork or housework.

domized to the treatment arms (intent-to-treat type analysis, whereby all patients who did not provide data were considered to have had nausea) confirmed the aforementioned results.

\section{Adverse events}

The mean symptom scores, as measured by the MDASI, for days 1 to 5 are recorded in Table 2 . There were no grade 3 or 4 toxicities attributable to the study drugs in any of the patients during the 5-day evaluation period. Patients who received olanzapine had significantly more drowsiness on day 2 compared with baseline and compared with the patients who received the FPD regimen (Table 2). Table 3 provides more detail of the grades of drowsiness on day 2 on the 2 study arms. The increased drowsiness resolved by day 3 , and was similar to day 1 (baseline) on days 3 , 4 , and 5 . No patients discontinued olanzapine because of excessive drowsiness or sedation, and there were no dose reductions for the olanzapine dose for any of the patients in the study. None of the other 16 evaluated symptoms had any significant changes from baseline. There were very few patients who experienced weight gain or glucose elevation from day 1 to day 5, and there was no difference in the study groups.

\section{Discussion}

The similar CR rates for the 2 regimens and improved nausea for the olanzapine regimen seen in this study are quite congruent with what was seen in a previous similar randomized, phase 3 trial in which chemotherapy naive patients, receiving HEC (cisplatin or an anthracycline plus cyclophosphamide), were randomized to a prophylactic antiemetic regimen of olanzapine, palonosetron, and dexamethasone or to a prophylactic regimen of aprepitant, palonosetron and dexamethasone. ${ }^{8}$ For the 121 patients receiving the olanzapine regimen and the 120 patients receiving the aprepitant regimen, there were no significant differences in $\mathrm{CR}$ rates in the acute, delayed, or overall periods. The number of patients with no nausea $(0$, scale $0-10$, visual analogue scale) was significantly higher in the delayed and overall periods for the patients who received the olanzapine regimen ${ }^{8}$.

The benefit of olanzapine for decreasing nausea has also been demonstrated in other phase 3 clinic trials. Tan and 
TABLE 3 Drowsiness scores on day 2 for the OPD and FPD study arms

\begin{tabular}{|lcc|}
\hline Score & $\begin{array}{c}\text { OPD regimen, } \\
\text { no. of patients } \\
(\mathbf{n}=\mathbf{5 1})\end{array}$ & $\begin{array}{c}\text { FPD regimen, } \\
\text { no. of patients } \\
(\mathbf{n}=\mathbf{5 0})\end{array}$ \\
\hline 0 & 3 & 14 \\
1 & 2 & 20 \\
\hline 2 & 9 & 14 \\
\hline 3 & 13 & 3 \\
\hline 4 & 6 & 2 \\
\hline 5 & 5 & 0 \\
\hline 6 & 3 & 0 \\
\hline 7 & 6 & 0 \\
\hline 8 & 4 & 0 \\
\hline 9 & 0 & 0 \\
\hline 10 & 0 & 0 \\
\hline
\end{tabular}

FPD, fosaprepitant, palonosetron, dexamethasone; OPD, olanzapine, palonosetron, dexamethasone

colleagues $^{7}$ studied the use of olanzapine as a prophylactic agent in patients receiving either moderately emetogenic chemotherapy (MEC), or HEC, by adding olanzapine to a prophylactic regimen of azasetron and dexamethasone in an unblinded, randomized phase 3 trial. In a total patient group of 229 patients receiving either MEC or HEC, $\mathrm{CR}$ and nausea control was significantly improved in the patients receiving olanzapine, azasetron, and dexamethasone compared with patients receiving azasetron and dexamethasone in the delayed and overall periods. Olanzapine improved the CR and nausea of the delayed period and overall period CINV and the quality of life in patients.

In another trial, this one having a randomized, double-blind, placebo-controlled design, ${ }^{20} 44$ patients scheduled to receive $\mathrm{MEC}$ or $\mathrm{HEC}$ received a 5-HT3 receptor antagonist, dexamethasone and a NK-1 receptor antagonist. Patients were then randomized to receive $5 \mathrm{mg}$ of olanzapine daily or placebo for 6 days beginning on the day before chemotherapy or placebo. CR rates and freedom from nausea were significantly improved in the patients receiving olanzapine.

A recently completed randomized, double-blind, phase 3 trial was performed using olanzapine for the prevention of CINV in chemotherapy-naive patients receiving cisplatin, $\geq 70 \mathrm{mg} / \mathrm{m}^{2}$ or cyclophosphamide-anthracycline-based chemotherapy, comparing olanzapine to placebo in combination with aprepitant, a 5-HT3 receptor antagonist, and dexamethasone. In this trial, complete freedom from nausea was the primary endpoint, and $\mathrm{CR}$ was a secondary endpoint. The olanzapine regimen was significantly more effective for the control of nausea and had a significantly higher CR rate in the acute, delayed, and overall periods. ${ }^{21}$

No nausea ( 0 on a scale of $0-10$, visual analogue scale) in the acute, delayed, and overall periods was a secondary endpoint in this study. This is in contrast to previous CINV prevention studies that have used "no significant nausea ( $<2.5$ on a scale of $0-10)$ " as a secondary endpoint. ${ }^{2,4-6,10}$ The "no nausea" measure seems to be an important objective assessment of the status of patients in the postchemotherapy period, possibly equally or more important than "complete remission," which has been used as the primary endpoint in most clinical trials. "No nausea" should be strongly considered for use as a primary endpoint in future clinical trials.

The benefit of olanzapine for decreasing nausea is in contrast to the control of nausea in clinical trials of the NK-1 receptor antagonists. Nausea has not been significantly improved by the use of fosaprepitant (aprepitant) in 2 phase 3 studies of patients receiving cisplatin ${ }^{22,23}$ and in 2 phase 3 studies of patients receiving an anthracycline and cyclophosphamide chemotherapy regimen. ${ }^{24,25}$ Two reviews on the prevention of chemotherapy-induced nausea concluded that $\mathrm{NK}-1$ receptor antagonists are not effective in controlling nausea. ${ }^{10,26}$

The data from the currently reported trial and the others, noted above, support National Comprehensive Cancer Network guidelines, which list the olanzapine, palonosetron, and dexamethasone regimen as an optional first-line therapy for the prevention of CINV in patients receiving $\mathrm{HEC}$ and $\mathrm{MEC}{ }^{27}$

There are economic benefits of olanzapine. Four days of generic oral olanzapine at $10 \mathrm{mg} / \mathrm{day}$, the dose used in this study and previous prophylactic studies, ${ }^{7-10}$ is about $\$ 3.00,{ }^{28}$ which is significantly lower than the cost of 1 day of intravenous fosaprepitant at $150 \mathrm{mg}$ (approximate wholesale acquisition cost: $\$ 257.00)$. $^{29}$

The nausea and vomiting observed in the current study was presumably because of both the HEC and the concurrent radiotherapy. It cannot be determined which of the treatment modalities contributed to more of the nausea and vomiting, although it is likely that the chemotherapy was most causative. The use of antiemetic agents previously used for the prevention of CINV caused by HEC seemed to be effective in the majority of patients receiving the combination chemotherapy radiotherapy. As suggested by the Multinational Association of Supportive Care in Cancer and the European Society of Medical Oncology radiotherapy guidelines, ${ }^{15}$ patients receiving chemotherapy radiotherapy should be given antiemetics based on the emetogenicity of the chemotherapy, unless the risk of emesis is higher with the radiotherapy than the chemotherapy.

The olanzapine seemed to be well tolerated in this trial. Patients who received olanzapine had more drowsiness on day 2 compared with baseline, but this resolved by day 3 despite continued oral olanzapine on days 3 and 4 , sug- 
gesting that patients adapted to the olanzapine. Because of the temporary drowsiness seen in this trial and reports of temporary drowsiness in some patients, more detailed data on drowsiness ratings should be obtained in future trials.

The current trial and supporting literature provide convincing evidence that olanzapine does decrease nausea and

\section{References}

1. Bloechl-Daum B, Deuson RR, Panagiotis M, et al. Delayed nausea and vomiting continue to reduce patients' quality of life after highly and moderately emetogenic chemotherapy despite antiemetic treatment. J Clin Oncol. 2006;24:4472-4478.

2. Navari RM. Management of chemotherapy-induced nausea and vomiting: Focus on newer agents and new uses for older agents. Drugs. 2013;73:249-262.

3. Navari RM. Palonosetron for the treatment of chemotherapy- induced nausea and vomiting. Expert Opin Pharmacother. 2014;15:2599-2608.

4. Aapro M, Carides A, Rapoport B. Aprepitant and fosaprepitant: a ten-year review of efficacy and safety. Oncologist. 2015;20:450-458.

5. Navari RM. Profile of netupitant/palonosetron fixed dose combination (NEPA) and its potential in the treatment of chemotherapyinduced nausea and vomiting (CINV). Drug Design, Development and Therapy. 2015;9:155-151.

6. Navari RM. Rolapitant for the treatment of chemotherapy induced nausea and vomiting. Expert Rev Anticancer Therapy. 2015;15:1127-1133.

7. Tan L, Liu J, Liu X, et al. Clinical research of olanzapine for prevention of chemotherapy-induced nausea and vomiting. J Exp Clin Cancer Res. 2009;28:1-7.

8. Navari RM, Gray SE, Kerr AC. Olanzapine versus aprepitant for the prevention of chemotherapy induced nausea and vomiting (CINV): a randomized phase III trial. J Supp Oncol. 2011;9:188-195.

9. Navari RM. Olanzapine for the prevention and treatment of chronic nausea and chemotherapy-induced nausea and vomiting. Eur J Pharmacol. 2014;722:180-186.

10. Navari RM. Treatment of chemotherapy induced nausea. Community Oncol. 2012;9:20-26.

11. Poon M, Dennis K, DeAngelis C, et al. A prospective study of gastrointestinal radiation therapy-induced nausea and vomiting. Support Care Cancer. 2014;22:1493-1507.

12. Vidall C, Fernandez-Ortega P, Cortinovis D, et al. Impact and management of chemotherapy/radiotherapy-induced nausea and vomiting and the perceptual gap between oncologists/oncology nurses and patients: a cross-sectional multinational survey. Support Care Cancer. 2015;23:3297-3305.

13. Ruhlmann CH, Iversen TZ, Okera M, et al. Multinational study exploring patients' perceptions of side effects induced by chemoradiotherapy. Radiother Oncol. 2015;117:333-337.

14. Dennis K, Poon M, Chow E. Nausea and vomiting induced by gastrointestinal radiation therapy: current status and future directions. Curr Opin Support Palliat Care. 2015;9:182-188.

15. Feyer PC, Maranzano E, Molassiotis A, et al. Radiotherapy-induced nausea and vomiting (RINV): MASCC/ESMO guideline for antiemetics in radiotherapy: update 2009. Support Care Cancer. 2011;19:S5-S14.

16. Dennis K, DeAngelis C, Jon F. et al. Aprepitant and granisetron for the prophylaxis of radiotherapy-induced nausea and vomiting after vomiting associated with chemotherapy with or without concomitant radiotherapy. Future investigations may include exploring the efficacy of olanzapine as an oral agent for the treatment of chronic nausea, unrelated to chemotherapy, as well as for clinical situations such as multiday chemotherapy or high-dose chemotherapy and stem-cell transplantation. moderately emetogenic radiotherapy for bone metastases: a prospective pilot study. Curr Oncol. 2014;21:760-767.

17. Yan M, Kumachev A, Siu LL, et al. Chemoradiotherapy regimens for locoregionally advanced nasopharyngeal carcinoma: A Bayesian network meta-analysis. Eur J Cancer. 2015;51:1570-1579.

18. Zhu L, Yuan L, Wang H, et al. A meta-analysis of concurrent chemoradiotherapy for advanced esophageal cancer. Plos One. 2015. doi: 10.137/journal.pone.0128616 June 5.

19. Cleeland CS, Mendoza TR, Wang XS, et al. Assessing symptom distress in cancer patients: The MD Anderson Symptom Inventory. Cancer. 2000;89:1634-1646.

20. Mizukami N, Yamanchi M, Koike K, et al. Olanzapine for the prevention of chemotherapy-induced nausea and vomiting in patients receiving highly or moderately emetogenic chemotherapy: a randomized, double-blind, placebo-controlled study. J Pain Symptom Manage. 2014;47:542-550.

21. Navari RM, Qin R, Ruddy KJ, et al. Olanzapine for the prevention of chemotherapy-induced nausea and vomiting (CINV) in patients receiving highly emetogenic chemotherapy (HEC): Alliance: A221301, a randomized, double-blind, placebo-controlled trial. ASCO Palliative Care Symposium, abstract 176, Boston, October, 2015.

22. Hesketh PJ, Grunberg SM, Gralla RJ. et al. The oral neurokinin-1 antagonist aprepitant for the prevention of chemotherapyinduced nausea and vomiting: A multinational, randomized, double-blind, placebo-controlled trial in patients receiving high-dose cisplatin - the Aprepitant Protocol 052 Study group. J Clin Oncol. 2003;21:4112-4119.

23. Hesketh PJ, Grunberg SM, Herrstedt J, et al. Combined data from two phase III trials of the NK-1 antagonist aprepitant plus a 5HT3 antagonist and a corticosteroid for prevention of chemotherapy- induced nausea and vomiting: Effect of gender on treatment response. Support Care Cancer. 2006;14:354-360.

24. Warr DG, Hesketh PJ, Gralla, RJ, et al. Efficacy and tolerability of aprepitant for the prevention of chemotherapy-induced nausea and vomiting in patients with breast cancer after moderately emetogenic chemotherapy. J Clin Oncol. 2005;23:2822-2830.

25. Rappport BL, Jordan K, Boice JA, et al. Aprepitant for the prevention of chemotherapy-induced nausea and vomiting associated with a broad range of moderately emetogenic chemotherapies and tumor types: a randomized, double-blind study. Support Care Cancer. 2010;18:423-431.

26. Ng TL, Hutton B, Clemons M. Chemotherapy-induced nausea and vomiting: Time for more emphasis on nausea? Oncologist. 2015;20:576-583.

27. National Comprehensive Cancer Network. Antiemesis: clinical practice guidelines in oncology. www.nccn.org. 2015. Accessed October 21, 2015.

28. Goodrx website. www.goodrx.com Accessed October 21, 2015.

29. The Medical Letter. 2015;57:60. 